\title{
Optimising age-replacement and extended non-renewing warranty policies in lifecycle costing
}

\author{
Shaomin $\mathrm{Wu}^{\star}$, Phil Longhurst
}

School of Applied Sciences, Cranfield University, Bedfordshire MK43 0AL, United Kingdom

Suggested Citation: Wu, S., Longhurst, P. Optimising age-replacement and extended non-renewing warranty policies in lifecycle costing (2011) International Journal of Production Economics, 130 (2), pp. 262-26\%.

Corresponding author. E-mail: s.m.wu@kent.ac.uk

\begin{abstract}
This paper analyses life cycle cost for equipment protected by both base and extended warranty policies from a consumer's perspective. We assume that the equipment have two types of failures: minor and catastrophic. A minor failure can be corrected by a minimal repair whereas a catastrophic failure can only be removed by a replacement. The equipment is assumed to be maintained at no charge to the consumer during the warranty period, whereas the consumer is fully charged for any maintenance on failures after the extended warranty expires. We formulate the expected life cycle cost of the equipment under a general failure time distribution, and then for special cases we prove that the optimal replacement and extended warranty policies exist to minimise the expected life cycle cost per unit time, which is examined by numerical examples.
\end{abstract}

Keywords: Life cycle cost; Warranty policy; Minimal repair; Opportunitybased replacement; Maintenance policy.

\section{Introduction}

The life cycle cost (LCC) of a piece of equipment is the summation of its cost estimates from inception to disposal. The objective of LCC analysis is to choose the most cost effective approach from a set of alternatives so that the optimum long-run ownership cost for the equipment is achieved. The basic elements of LCC may involve initial capital costs, operation costs, 
maintenance costs and disposal costs. Recently, LCC has been studied by many authors (Chung and Wee, 2008; Ahiska and King, 2010; Kleyner and Sandborn, 2008; Mascle and Zhao, 2008).

Reliability is one of the most important factors influencing the LCC of a piece of equipment as it is associated with many elements of the LCC. For example, a piece of equipment with higher reliability may involve higher initial capital costs, lower operation costs, lower maintenance costs due to fewer failures and longer operation time, and may also incur lower disposal costs because of potential for reuse or resale. According to British Standard (1997), costs associated with equipment safety, reliability, maintainability and maintenance support performance, which are not that apparent but need to be accounted in LCC models, may include the following three elements, as appropriate: (1) Unavailability costs: including maintenance costs and costs associated with loss of equipment function, such as reduced productivity; (2) Warranty costs: for warranty-type agreements, and (3) Liability costs: costs of liabilities due to equipment failure and their injurious effects needs to be considered as part of the LCC.

Maintenance and warranty policies that influence the above three costs should therefore be considered in LCC analysis.

From a consumer's perspective, maintenance costs may vary with different maintenance policies. In past decades, a huge number of maintenance policies have been proposed. More detailed discussion on maintenance policies can be found in Wang (2002) and Wu and Zuo (2010). Among the existing maintenance policies, a commonly used policy is a combination of corrective maintenance and opportunity-based age replacement: a piece of equipment is repaired upon a minor failure, and replaced by a new identical one at a predetermined age or on a catastrophic failure. This maintenance policy is usually adopted in many industries such as maintaining building services systems.

Warranty is also an important driver influencing maintenance costs and warranty costs. In a time order when a warranty policy is performed, it can be either a base warranty or a combination of a base and an extended warranties. An extended warranty may cover maintenance cost for equipment after its manufacturer's base warranty expires. The length of the extended warranty is important from a consumer's perspective. A long extended warranty may be more costly when equipment is purchased, but it can be more cost-effective in the long run as any replacements during the extended warranty will be 
served by the warranty provider.

In this study, we assume an opportunity-based age replacement. That is: there are two types of failure: type I and type II failures; type I failure is a minor failure which can be corrected by a minimal repair whereas type II failure is a catastrophic failure that can only be corrected by a replacement. We also assume that the length of the extended warranty policies is available for selection. Under these assumptions, we optimise the opportunity-based age replacement policy and the length of the extended warranty to minimise the expected life cycle cost per unit time.

The paper is structured as follows. Section 2 reviews the existing literature on maintenance policy optimisation considering warranty. Section 3 formulates the expected life cycle cost and the expected length of a life cycle. Section 4 is a discussion section. Section 5 offers numerical examples to validate the derived models. The last section concludes with findings.

\section{Prior work}

There is an increasing amount of work on the optimisation of maintenance policies for equipment covered by warranty contracts. For a review of related research in this area, the reader is referred to Murthy and Djamaludin (2002). Publications appeared from year 2006 to year 2010 is briefly reviewed below.

Maintenance policy optimisation that considers warranties is studied by some authors. For example, Jack and Murthy (2007) investigate optimal pricing strategies for the extended warranty provider and optimal maintenance and replacement strategies for the consumer. Wu and Li (2007), and Wu and Xie (2008) develop warranty cost models for repairable and non-repairable equipment with a dormant state, respectively. Yun et al. (2008) investigate two warranty servicing strategies involving minimal and imperfect repairs, where the two strategies differ as to whether the effectiveness of imperfect repair is dependent upon equipment age. Chien (2010) optimises age-replacement policies for equipment under a new warranty strategy, where he combines a fully renewable free replacement with a pro-rata warranty policy. Chen and Chien (2007) consider maintenance policy optimisation for equipment covered by warranty contracts and assume that the equipment have both type I and type II failures, and derive cost models and examine the effects of three 
PM options on the cost from both the manufacturer's and buyer's perspectives whilst assuming that the equipment are sold under a free-replacement renewing warranty. Jack et al. (2009) considers a maintenance policy during the post-warranty period under the renewing warranty policy, here the life cycle is defined from the user's perspective.

Having reviewed prior work one finds that little discussion has been made on the combination of extended warranty policies and opportunity-based replacement policies, while such a combination is frequently used in practice, for example, in maintaining building services systems.

\section{Problem formulation}

There are a number of different warranty policies that have been studied (Blischke and Murthy, 1994). In this paper, we consider a NFRW (nonrenewing free repair warranty) policy. Under an NFRW policy, the manufacturer guarantees a satisfactory service only during the base warranty period and the failed equipment is replaced by the manufacturer at no cost to the user (NFRW) during the base warranty period. A typical scenario of the different periods in the life cycle is shown in Figure 1.

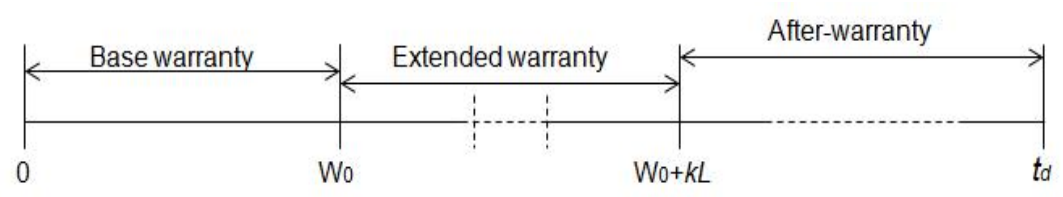

Fig. 1. The life cycle span

\subsection{Assumptions and notation}

As above-mentioned, we consider an opportunity-based age replacement policy as follows. The following assumptions are held.

- The equipment starts from time $t=0$. The base warranty period is $\left(0, w_{0}\right]$ and the extended warranty period is $\left(w_{0}, w\right]$, where $w=w_{0}+k L, L$ is a given time period, and $k(=0,1,2, \ldots)$ can be optimised.

- The equipment has two types of possible failures at age $t$ : minor failure (or type I failure) and catastrophic failure (or type II failure). A type I failure occurs with probability $q(t)$ and can be corrected with minimal repair that 
restores the equipment in the state it was before failure. A type II failure occurs with probability $1-q(t)$ and can only be corrected by replacement.

- Within the warranty time $(0, w)$, upon failures, the equipment is corrected with either minimal repair or replacement. After the extended warranty expires, the equipment is replaced either at pre-specified time $t_{d}$ (where $\left.t_{d}>w\right)$ or upon type II failure, whichever occurs first.

- The equipment is maintained, for both minimal repair and replacement, at no charge to the consumer during the base and extended warranty periods. Whereas the consumer is fully charged for any maintenance when a failure, which can be type I failure or type II failure, occurs after the extended warranty expires.

- The following costs are assumed.

- $c_{\mathrm{I}}$ and $C_{\mathrm{I}}$ are the repair coss per type I failure and the total repair costs on type I failure after the extended warranty expires, respectively;

- $c_{\mathrm{II}}$ and $C_{\mathrm{II}}$ are the replacement cost per type II failure and the total replacement cost on type II failure after the extended warranty expires, respectively;

- $c_{f}$ and $C_{f}$ are the unavailability and liability costs incurred on a type I or type II failures, and the total unavailability and liability costs on type I or type II failures, respectively;

- $c_{e}$ and $C_{e}$ are the cost of purchasing extended warranty for a time period $L$, and the total warranty cost from a consumer's perspective, respectively;

- $C_{0}$ and $c_{d}$ are the initial capital and the cost per replacement at time $t_{d}$, respectively.

- Both the base warranty and the extended warranty are NFRW.

- Time on either minimal repair or replacement is negligible.

\subsection{Model development}

An optimal LCC may be chosen based on the following optimal approach:

- Minimising LCC by choosing the optimal number of the extended warranty periods $k$ and the optimal design life $t_{d}$.

We derive the following life cycle cost. For other warranty policies, similar results can be obtained by using the following derivation approach. 
The expected cost incurred during the life cycle can be defined as

$$
C\left(k, t_{d}\right)=C_{0}+C_{e}+E\left[C_{\mathrm{I}}\right]+E\left[C_{\mathrm{II}}\right]+E\left[C_{f}\right],
$$

where $E[\bullet]$ represents the expected value.

As the base warranty is an NFRW, the cost charged to the consumer for repair (due to type I failure) or replacement (due to type II failure) within the base warranty period $\left(0, w_{0}\right]$ is 0 . The extra cost for purchasing the extended warranty with length of $k L$ is $k c_{e}$. Hence, from a consumer's perspective, the total cost on the extended warranty within $(0, w)$ is given by

$$
C_{e}=k c_{e} .
$$

Denote $h(t)$ as the naked hazard function of the equipment. According to Block et al. (1985), the cumulative distribution function of time to type I failures, $x$, is given by $F_{\mathrm{I}}(x)=1-\exp \left\{-\int_{0}^{x} q(t) h(t) d t\right\}$ and the lifetime distribution between successive type II failures, is $F_{\mathrm{II}}(z)=1-\exp \left\{-\int_{0}^{z}(1-q(t)) h(t) d t\right\}$.

Let $z_{i}$ be the time between the $(i-1)$-th and the $i$-th type II failures and $S_{n}=$ $\sum_{i=1}^{n} z_{i}\left(S_{0}=0\right.$, by convention). Denote $N(t)$ as the number of replacements within time interval $(0, t)$ so that $N(t)=\max \left\{n: S_{n}<t\right\}$. Then, $\{N(t), t \geq$ $0\}$ is a renewal process, $\operatorname{Pr}(N(t)=i)=F_{\mathrm{II}}^{(i)}(t)-F_{\mathrm{II}}^{(i+1)}(t)$ and the expected value of $N(t)$ is given by $M(t)=E(N(t))=\sum_{i=1}^{\infty} F_{\mathrm{II}}^{(i)}(t)$, where $M(t)$ is the renewal function, and $F_{\mathrm{II}}^{(i)}(t)$ is the $i$-fold convolution of $F_{\mathrm{II}}(t)$ with $F_{\mathrm{II}}^{(0)}(t)=$ 1. $M(t)$ can also be re-written as

$$
M(t)=F_{\mathrm{II}}(t)+\int_{0}^{t} M(t-x) d F_{\mathrm{II}}(x) .
$$

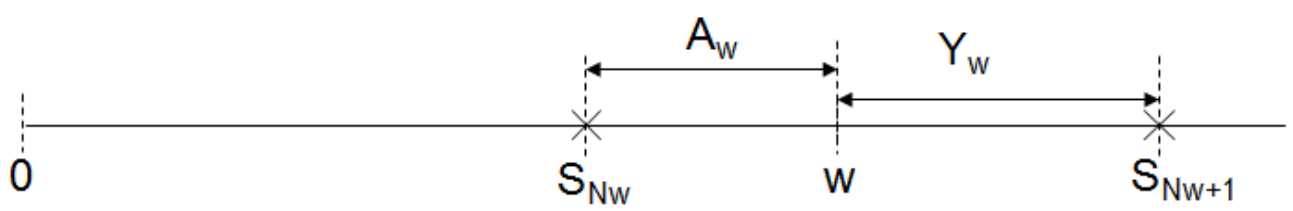

Fig. 2. Excess of the renewal process

Denote $N_{w}=\max \left\{n: S_{N_{w}}<w\right\}$, and $S_{N_{w}}=\sum_{i=1}^{N(w)} z_{i}$. The relationship among $S_{N_{w}}, w\left(=w_{0}+k L\right)$, and $S_{N_{w}+1}$ is shown in Figure 2. Denote $A_{w} \stackrel{\text { def }}{=}$ $w-S_{N_{w}}$, and $Y_{w} \stackrel{\text { def }}{=} S_{N_{w}+1}-w$. Then, $A_{w}$ is the current lifetime at time $w$, and $Y_{w}$ is the excess lifetime at time $w$, here both $A_{w}$ and $Y_{w}$ are lifetimes 
to type II failures. According to Karlin and Taylor (2007), the distribution function of $A_{w}$ is given by

$$
H_{w}(y)=\operatorname{Pr}\left(A_{w}<y\right)=F_{\mathrm{II}}(w)-\int_{0}^{w-y}\left(1-F_{\mathrm{II}}(w-t)\right) d M(t),
$$

The distribution function of $Y_{w}$ is given by

$$
G_{w}(y)=\operatorname{Pr}\left(Y_{w}<y\right)=F_{\mathrm{II}}(w+y)-\int_{0}^{w}\left(1-F_{\mathrm{II}}(w+y-t)\right) d M(t)
$$

On the other hand, given that the equipment has survived $A_{w}=w-S_{N_{w}}$ time units, the distribution of time to the first type I failure is

$$
\begin{aligned}
G_{\mathrm{I}}(x) & =\operatorname{Pr}\left(X_{\mathrm{I}}<x+w-S_{N_{w}} \mid X_{\mathrm{I}}>w-S_{N_{w}}\right) \\
& =\frac{\operatorname{Pr}\left(X_{\mathrm{I}}-A_{w}<x\right)-\operatorname{Pr}\left(X_{\mathrm{I}}-A_{w}<0\right)}{1-\operatorname{Pr}\left(X_{\mathrm{I}}-A_{w}<0\right)} \\
& =\frac{\int_{0}^{\infty} F_{\mathrm{I}}(x+y) d H_{w}(y)-\int_{0}^{\infty} F_{\mathrm{I}}(y) d H_{w}(y)}{1-\int_{0}^{\infty} F_{\mathrm{I}}(y) d H_{w}(y)}
\end{aligned}
$$

Denote $h_{\mathrm{I}}(x)=\frac{1}{1-G_{\mathrm{I}}(x)} \frac{d G_{\mathrm{I}}(x)}{d x}$. Let $t_{0}=t_{d}-w$. Then the probability of the event that no type II failure occurs within time interval $\left(w, t_{d}\right)$ is $\bar{G}_{w}\left(t_{0}\right)$, where $\bar{G}_{w}(t)=1-G_{w}(t)$. Then we have: (1) the number of type I failures is $\bar{G}_{w}\left(t_{0}\right) \int_{0}^{t_{0}} h_{\mathrm{I}}(t) d t$, given the event that no type II failure occurs within time interval $\left(w, t_{d}\right)$; and (2) the number of type I failures is $\int_{0}^{t_{0}} h_{\mathrm{I}}(t) d G_{w}(t)$, given the event that type II failures occur within time interval $\left(w, t_{d}\right)$.

Thus, the expected cost on minimal repairs for type I failures after the extended warranty period expires is given by

$$
E\left[C_{\mathrm{I}}\right]=c_{\mathrm{I}} \bar{G}_{w}\left(t_{0}\right) \int_{0}^{t_{0}} h_{\mathrm{I}}(t) d t+c_{\mathrm{I}} \int_{0}^{t_{0}} h_{\mathrm{I}}(t) d G_{w}(t)
$$

Similarly, the expected replacement costs incurred due to type II failures after the extended warranty expires is

$$
E\left[C_{\mathrm{II}}\right]=c_{d} \bar{G}_{w}\left(t_{0}\right)+c_{\mathrm{II}} G_{w}\left(t_{0}\right)=c_{d}+\left(c_{\mathrm{II}}-c_{d}\right) G_{w}\left(t_{0}\right)
$$


$C_{f}$ is the unavailability and liability cost incurred on a type I or type II failures within the life cycle including both the warranty period the after warranty period. Then we have the following Lemma.

The proofs of the Lemmas in this paper can be found in the Appendix.

Lemma $1 E\left[C_{f}\right]$ is given by

$$
\begin{aligned}
E\left[C_{f}\right] & =c_{f} M(w) \int_{0}^{w} \int_{0}^{x} q(t) h(t) d t d F_{\mathrm{II}}(x) \\
& +c_{f}\left(\int_{0}^{w} \int_{0}^{y} q(t) h(t) d t d H_{w}(y)+\bar{G}_{w}\left(t_{0}\right) \int_{0}^{t_{0}} h_{\mathrm{I}}(t) d t+\int_{0}^{t_{0}} h_{\mathrm{I}}(t) d G_{w}(t)\right) \\
& +c_{f}\left(1-F_{\mathrm{II}}(w)\right) \int_{0}^{w} q(t) h(t) d t+c_{f}\left(M(w)+G_{w}\left(t_{0}\right)\right)
\end{aligned}
$$

In sum, the expected LCC is therefore given by

$$
\begin{aligned}
C\left(k, t_{d}\right) & =C_{0}+C_{e}+E\left[C_{\mathrm{I}}\right]+E\left[C_{\mathrm{II}}\right]+E\left[C_{f}\right] \\
& =C_{0}+k c_{e}+c_{d}+\left(c_{\mathrm{II}}-c_{d}\right) G_{w}\left(t_{0}\right)+c_{f}\left(M(w)+G_{w}\left(t_{0}\right)\right) \\
& +\left(c_{\mathrm{I}}+c_{f}\right) \bar{G}_{w}\left(t_{0}\right) \int_{0}^{t_{0}} h_{\mathrm{I}}(t) d t+\left(c_{\mathrm{I}}+c_{f}\right) \int_{0}^{t_{0}} h_{\mathrm{I}}(t) d G_{w}(t)+c_{f}\left(1-F_{\mathrm{II}}(w)\right) \int_{0}^{w} q(t) h(t \\
& +c_{f} M(w) \int_{0}^{w} \int_{0}^{x} q(t) h(t) d t d F_{\mathrm{II}}(x)+c_{f} \int_{0}^{w} \int_{0}^{y} q(t) h(t) d t d H_{w}(y)
\end{aligned}
$$

After the extended warranty expires, the equipment is replaced by a new identical one upon type II failure or at the fixed design life time $t_{d}$, whichever comes first. The replacement time length $Y$ will be $Y(w)$ or $t_{0}$ depending on whether the lifetime is shorter than $t_{0}$ or not. That is $Y=\min \left\{Y(w), t_{0}\right\}=$ $\left\{\begin{array}{l}Y(w) t \in\left[0, t_{0}\right) \\ t_{0} \quad t \in\left[t_{0}, \infty\right)\end{array}\right.$. The expected length of a replacement cycle after the extended warranty expires is given by

$$
E(Y)=E\left[\min \left\{Y(w), t_{0}\right\}\right]=\int_{0}^{t_{0}} t d G_{w}(t)+t_{0}\left(1-G_{w}\left(t_{d}\right)\right)
$$




$$
=\int_{0}^{t_{0}}\left(1-G_{w}(t)\right) d t=\int_{0}^{t_{0}} \bar{G}_{w}(t) d t
$$

The expected length of a total life cycle span is given by

$$
B\left(k, t_{d}\right)=w+\int_{0}^{t_{0}} \bar{G}_{w}(t) d t
$$

Let $D\left(k, t_{d}\right)$ signify the expected life cycle cost per unit time, then we have

$$
D\left(k, t_{d}\right)=\frac{C\left(k, t_{d}\right)}{B\left(k, t_{d}\right)}
$$

\section{Discussion}

The cost $c_{d}$ of replacement at time $t_{d}$ is assumed to be a constant value in the above discussion. However, $c_{d}$ might depend on $t_{d}-S_{N_{w}}$, which is the equipment age. If the replacement after the extended warranty expires because the equipment reaches its design lifetime $t_{d}$, but not due to type II failure, the equipment can be re-sold. Thus, the re-sale price might depend on the equipment age, which is $t_{d}-S_{N_{w}}$. Discussion on assessing resale price or salvage price in reliability and maintenance can be found in Monga and Zuo (2001).

In the preceding sections, repair upon type I failures is assumed to be minimal repair and time on repair is negligible. Obviously, repair effects can be other types, for example, imperfect repair (see Wu and Clements-Croome (2005), for example) can be considered.

For the expected life cycle cost per unit time given in Eq. (13), the following special cases are also worthwhile mentioning.

\subsection{Special cases}

Case 1. If $q(t)=1$, then the maintenance policy discussed in the preceding section is a classical periodic preventive replacement policy with minimal repair upon failure. In this case, we have $F_{\mathrm{II}}(t)=0, N_{w}=0, S_{N_{W}}=0$, $M(w)=0, H_{w}(t)=0, G_{w}(t)=0, G_{\mathrm{I}}(x)=\frac{F_{\mathrm{I}}(x+w)-F_{\mathrm{I}}(w)}{1-F_{\mathrm{I}}(w)}$, and $h_{\mathrm{I}}(x)=$ 
$h(x+w)$. Then $C\left(k, t_{d}\right)=C_{0}+k c_{e}+c_{d}+c_{\mathrm{I}} \int_{w}^{t_{d}} h(t) d t+c_{f} \int_{0}^{t_{d}} h(t) d t$ and $B\left(k, t_{d}\right)=t_{d}$. The expected life cycle cost per unit time is therefore given by

$$
D\left(k, t_{d}\right)=\frac{C\left(k, t_{d}\right)}{B\left(k, t_{d}\right)}=\frac{C_{0}+k c_{e}+c_{d}+c_{\mathrm{I}} \int_{w}^{t_{d}} h(t) d t+c_{f} \int_{0}^{t_{d}} h(t) d t}{t_{d}}
$$

Case 2. If $q(t)=0$, then the maintenance policy discussed in the preceding section is a classical age-based replacement policy. In this case, we have $F_{\mathrm{I}}(t)=0$, and $h_{\mathrm{I}}(t)=0$. The expected life cycle cost per unit time is therefore given by

$$
D\left(k, t_{d}\right)=\frac{C_{0}+k c_{e}+c_{d}+\left(c_{\mathrm{II}}-c_{d}\right) G_{w}\left(t_{0}\right)+c_{f}\left(M(w)+G_{w}\left(t_{0}\right)\right)}{w+\int_{0}^{t_{0}} \bar{G}_{w}(t) d t}
$$

Case 3. Assume $q(t)=q_{0}$ (where $\left.q_{0} \in[0,1]\right)$ and $h(t)=\lambda$. Then, $F_{\mathrm{I}}(x)=$ $1-e^{-q_{0} \lambda x}, F_{\mathrm{II}}(x)=H_{w}(x)=G_{w}(x)=1-e^{-\left(1-q_{0}\right) \lambda x}, M(t)=\left(1-q_{0}\right) \lambda t$, and $h_{\mathrm{I}}(x)=q_{0} \lambda$. Denote $C_{1}=c_{\mathrm{II}}+c_{f}-c_{d}+\left(c_{\mathrm{I}}+c_{f}\right) q_{0} \lambda$ and $\psi(k)=$ $C_{0}+C_{1}+k c_{e}+c_{d}+c_{f}\left(\lambda w+\frac{q_{0}}{1-q_{0}}\right)-c_{f} q_{0}\left(\left(1-q_{0}\right) \lambda^{2} w^{2}+\lambda w+\frac{1}{1-q_{0}}\right) e^{-\left(1-q_{0}\right) \lambda w}$, then we have

$$
C\left(k, t_{d}\right)=\psi(k)+\left(c_{\mathrm{I}}+c_{f}\right) q_{0} \lambda t_{0} \bar{G}_{w}\left(t_{0}\right)-C_{1} \bar{G}_{w}\left(t_{0}\right)
$$

and

$$
B\left(k, t_{d}\right)=w+\frac{1}{\left(1-q_{0}\right) \lambda}-\frac{1}{\left(1-q_{0}\right) \lambda} \bar{G}_{w}\left(t_{0}\right)
$$

The parameter setting in Case 3 is widely adopted in practice because most industries assume constant failure rates of their equipment.

Case 4 It is natural in commercial industries that the cost of replacement could be dependent on design time and the age of product. As such, costs, including $c_{f}, c_{\mathrm{I}}, c_{\mathrm{II}}$ and $c_{d}$ in Eqs. (10) and (12), can be extended to be time-depended.

Other cases If $t_{0}=0$, then the warranty covers the whole life time until the design life $t_{d}$ is reached. If $k=0$, then no extended warranty is purchased. In this case, we also have $w=w_{0}$.

\subsection{Optimal policy for the special cases}

In this section, we seek to optimise a combination of $k$ and $t_{d}$ that minimises $A\left(k, t_{d}\right)$. 
If we substitute the numerator and the denominator of Eq. (13) with $C\left(k, t_{d}\right)$ from Eq. (10) and $B\left(k, t_{d}\right)$ from Eq. (12), respectively, we may find that the expression is very complex. In practice, if $L=1$ (year), the value $k$ has only several possible values, for example, $k=1,2, \ldots 10$. We can therefore fix the maximum value $k=k_{\max }$, and optimise $B\left(k_{0}, t_{d}\right)$, as shown in Table 1 .

In practice, given a fixed time period $L$, the number $k_{0}$ of the extended warranty periods has only limited possibilities. The design life $t_{d}$ might also have a limited number of solutions, it can be easy to obtain the optimal solutions based on Table 1 . For example, given $L=6$ months, then $k_{0}$ might smaller than 100 and $t_{d}$ might not exceed 600 months.

Table 1

Optimal solution $\left(k^{*}, t_{d}^{*}\right)$

Inputs: $h(t), q(t), w_{0}, L, c_{\mathrm{I}}, c_{\mathrm{II}}, c_{d}, c_{f}, c_{e}, k_{\max } ;$ Outputs: $k^{*}, t_{d}^{*}$

1: for $k_{0} \in\left\{1, \ldots, k_{\max }\right\}$ do

2: $\quad$ compute $F_{\text {II }}$ as defined by $F_{\mathrm{II}}(z)$

3: compute $H_{w}(y)$ and $G_{w}(y)$ as defined by Eq. (4) and Eq. (5), respectively

4: $\quad$ compute $C\left(k, t_{d}\right)$ and $B\left(k, t_{d}\right)$ defined by Eq. (10) and Eq. (12), respectively

5: $\quad$ find the optimal $T_{k_{0}}^{*}$ to minimise $D\left(k_{0}, t_{d}\right)$ defined by Eq. (13), where $w+k L<t_{d}$

6: end

7: let $T_{k_{m}}^{*}=\min \left\{T_{k_{0}}^{*}, \ldots, T_{k_{\max }}^{*}\right\}, t_{d}^{*}=T_{k_{m}}^{*}$, and $k^{*}=k_{m}$

Based on the above discussions, for special cases, we can prove the existence of the optimal solutions as follows.

Lemma 2 For a fixed $k=k_{0}$, if $q(t)=1$,

$$
\left(c_{\mathrm{I}}+c_{f}\right) h(w) w-C_{0}-k c_{e}-c_{d}-c_{f} \int_{0}^{w} h(t) d t<0,
$$

and $h(t)$ is a non-decreasing positive function, then there exists one solution $t_{d}^{*}$ that minimises the life cycle average cost in Eq. (14).

Lemma 3 For a fixed $k=k_{0}$, if $q(t)=0, c_{\mathrm{II}}-c_{d}+c_{f}>0$, and

$$
g_{w}(0)\left(c_{\mathrm{II}}-c_{d}+c_{f}\right) w-\left(C_{0}+k_{0} c_{e}+c_{d}+c_{f} M(w)\right)<0
$$

where $g_{w}(0)=\left.\frac{d G_{w}\left(t_{d}-w\right)}{d t_{d}}\right|_{t_{d}=w}$, then there exists one solution $t_{d}^{*}$ which minimises the life cycle average cost in Eq. (15). 
Table 2

Parameter setting

\begin{tabular}{cccccccccc}
\hline$w_{0}$ & $L$ & $c_{0}$ & $c_{e}$ & $c_{d}$ & $c_{\mathrm{I}}$ & $c_{\mathrm{II}}$ & $c_{f}$ & $\alpha$ & $\gamma$ \\
3.6 & 1.2 & 0 & 5 & 150 & 40 & 100 & 70 & 30 & 2 \\
\hline
\end{tabular}

Table 3

Optimisation of the design time $t_{d}$.

\begin{tabular}{c|cccccccccc}
\hline \hline$t_{d}$ & 7 & 8 & 9 & 10 & 11 & 12 & 13 & 14 & 15 & 16 \\
\hline$D\left(k, t_{d}\right)$ & 25.07 & 22.66 & 21.03 & 19.96 & 19.30 & 18.96 & $\underline{\mathbf{1 8 . 8 8}}$ & 19.00 & 19.29 & 19.74 \\
\hline \hline$t_{d}$ & 17 & 18 & 19 & 20 & 21 & 22 & 23 & 24 & 25 & 26 \\
\hline$D\left(k, t_{d}\right)$ & 20.30 & 20.98 & 21.74 & 22.59 & 23.51 & 24.49 & 25.53 & 26.60 & 27.72 & 28.88 \\
\hline \hline
\end{tabular}

Lemma 4 For a fixed $k=k_{0}$, the optimal design time $t_{d}$ is given by

$$
t_{d} \approx \frac{-\beta_{2}+\sqrt{\beta_{2}^{2}-4 \beta_{1} \beta_{3}}}{2 \beta_{1}}+w
$$

where $\beta_{1}=\frac{1}{2}\left(c_{\mathrm{I}}+c_{f}\right)\left(1-q_{0}\right) q_{0} \lambda^{2}, \beta_{2}=-\left(c_{\mathrm{I}}+c_{f}\right) q_{0} \lambda+\left(c_{\mathrm{I}}+c_{f}\right)[1+(1-$ $\left.\left.q_{0}\right) \lambda w\right] q_{0} \lambda, \beta_{3}=-\left(c_{\mathrm{I}}+c_{f}\right) q_{0} \lambda w-C_{1}\left(1-q_{0}\right) \lambda w-C_{1}-\psi\left(k_{0}\right)$.

\section{$5 \quad$ Numerical experiments}

In optimising the above problems, we need to approximate the renewal function given in Eq. (3). Here, we use the approached developed by Xie (1989) for approximating renewal functions. Assume the failure time distribution of the equipment under study is Weibull distribution, i.e., $h(t)=\frac{\gamma}{\alpha}\left(\frac{t}{\alpha}\right)^{\gamma-1}$, where $\gamma>1$. Without loss of generality, we assume $q(t)$ is constant.

The parameters in Table 2 are typical examples. In practice, the parameters set in Case 3 might be used as most industries set the failure rates as constant.

\subsection{Optimisation of the design life when $w$ is fixed}

Set parameters as shown in Table 2 and $q(t)=0.98$. Let $k_{0}=2$, which implies that the length $k_{0}$ of the extended warranty is fixed and only the design lifetime $t_{d}$ will be optimised. In this case, if we use the algorithm listed in Table 1 , we can find when $t_{d}=13, D\left(k, t_{d}\right)$ reaches its minimum value 18.88. See Table 3 for details. 


\begin{tabular}{c|ccccccccc}
\hline \hline$k_{0}$ & 0 & 1 & 2 & 3 & 4 & 5 & 6 & 7 & 8 \\
\hline$t_{d}$ & 8.6 & 9.8 & 11.0 & 12.2 & 13.4 & 14.6 & 15.8 & 17.0 & 18.2 \\
\hline$D\left(k, t_{d}\right)$ & 21.46 & 20.17 & 19.30 & 18.71 & 18.31 & 18.05 & 17.88 & 17.79 & 17.74 \\
\hline \hline$k_{0}$ & 9 & 10 & 11 & 12 & 13 & 14 & 15 & 16 & \\
\hline$t_{d}$ & 19.4 & 20.6 & 21.8 & 23.0 & 24.2 & 25.4 & 26.6 & 27.8 & \\
\hline$D\left(k, t_{d}\right)$ & $\underline{\mathbf{1 7 . 7 3}}$ & 17.74 & 17.76 & 17.78 & 17.81 & 17.83 & 17.84 & 17.86 & \\
\hline \hline
\end{tabular}

5.2 Optimisation of the length of the warranty period and the design life

Set parameters as shown in Table 2 , and $q(t)=0.98$. We are trying to optimise both the length of warranty period and the design life. If we use the algorithm listed in Table 1 , we can find when $t_{d}=19.4$ and $k_{0}=9, D\left(k, t_{d}\right)$ reaches the optimum value of 17.73 . See Table 4 for details.

\subsection{Parameter $q(t)$}

If $q(t)$ changes, and we optimise $t_{d}$ but keep $k_{0}$ constant, it is found that the optimal $t_{d}$ remains unchanged. For example, if we set parameters as shown in Table 2 , set $k_{0}=1$, and change $q(t)$ from 0.02 to 0.5 with step 0.02 , it is found that the optimal value of $t_{d}=12.80$ and it remains changed .

\section{Conclusions}

There is an increasing trend of encouraging consumers to buy extended warranty contracts. As both the length of warranty period and time to replacement influence the life cycle cost of equipment, selection of the extended warranty period and replacement policies is therefore important from a consumer's perspective.

In practice, a piece of equipment is usually repaired after a minor failure occurs; and it is replaced by a new identical one after a catastrophic failure occurs. This paper formulated the expected life cycle cost considering policies for an extended warranty and maintenance that are: opportunity-based age replacement policy with minimal repair. Conditions for the existence of 
optimal solutions for both the length of the extended warranty period and the design life for special cases are offered and proved.

\section{Acknowledgment}

I am grateful to the three anonymous referees for their valuable comments and suggestions that improved the quality of this paper. This research is supported by Engineering and Physical Sciences Research Council (EPSRC) of the United Kingdom (EPSRC Grant reference: EP/G039674/1).

\section{References}

Ahiska, S. S., King, R. E., 2010. Life cycle inventory policy characterizations for a single-product recoverable system. International Journal of Production Economics 124 (1).

Blischke, W. R., Murthy, D. N. P., 1994. Warranty Cost Analysis. Marcel Dekker, New York.

Block, H. W., Borges, W. S., Savits, T. H., 1985. Age-dependent minimal repair. Journal of Applied Probability 22 (2), 370-385.

British Standard, 1997. Reliability of systems, equipment and componentspart 23: Guide to life cycle costing, BS-5760-23.

Chen, J., Chien, Y., 2007. Renewing warranty and preventive maintenance for products with failure penalty post-warranty. Quality and Reliability Engineering International 23 (1), 107-121.

Chien, Y., 2010. Optimal age for preventive replacement under a combined fully renewable free replacement with a pro-rata warranty. International Journal of Production Economics 124 (1), 198-205.

Chung, C., Wee, H., 2008. Green-component life-cycle value on design and reverse manufacturing in semi-closed supply chain. International Journal of Production Economics 113 (2), 528-545.

Jack, N., Iskandar, B. P., Murthy, D. N. P., 2009. A repair-replace strategy based on usage rate for items sold with a two-dimensional warranty. Reliability Engineering and System Safety 94 (2), 611-617.

Jack, N., Murthy, D. N. P., 2007. A flexible extended warranty and related optimal strategies. Journal of the Operational Research Society 58 (12), $1612-1620$. 
Karlin, S., Taylor, H. M., 2007. A First Course in Stochastic Processes. Academic, New York.

Kleyner, A., Sandborn, P., 2008. Minimizing life cycle cost by managing product reliability via validation plan and warranty return cost. International Journal of Production Economics 112 (2), 796-807.

Mascle, C., Zhao, H. P., 2008. Integrating environmental consciousness in product/process development based on life-cycle thinking. International Journal of Production Economics 112 (1), 5-17.

Monga, A., Zuo, M. J., 2001. Optimal design of series-parallel systems considering maintenance and salvage value. Computers and Industrial Engineering 40 (4), 323-337.

Murthy, D. N. P., Djamaludin, I., 2002. New product warranty: A literature review. International Journal of Production Economics 79 (3), 231-260.

Wang, H., 2002. A survey of maintenance policies of deteriorating systems. European Journal of Operational Research 139 (3), 469-489.

Wu, S., Clements-Croome, D., 2005. Preventive maintenance models with random maintenance quality. Reliability Engineering and System Safety 90 (1), 99-105.

Wu, S., Li, H., 2007. Warranty cost analysis for products with a dormant state. European Journal of Operational Research 182 (3), 1285-1293.

Wu, S., Xie, M., 2008. Warranty cost analysis for nonrepairable services products. International Journal of Systems Science 39 (3), 279-288.

Wu, S., Zuo, M. J., 2010. Linear and nonlinear preventive maintenance models. IEEE Transactions on Reliability 59 (1), $242-249$.

Xie, M., 1989. On the solution of renewal-type integral equations. Communications in Statistical Simulation 18 (1), 281-293.

Yun, W. Y., Murthy, D. N. P., Jack, N., 2008. Warranty servicing with imperfect repair. International Journal of Production Economics 111 (1), 159169.

\section{Appendix}

\section{Proof of Lemma 1.}

- If the number of type II failures within time interval $(0, w+k L)$ is $N(w)$, then the number of minor failures is given by $\sum_{i=1}^{N(w)} \int_{0}^{z_{i}} q(t) h(t) d t$, where $z_{i}$ is time to type II failure. The expected number of minor failures within time interval $\left(0, S_{N_{w}}\right)$ is given by 


$$
\begin{aligned}
E\left[\sum_{i=1}^{N(w)} \int_{0}^{z_{i}} q(t) h(t) d t\right] & =\sum_{i=1}^{\infty}\left\{\operatorname{Pr}\{N(w)=i\} \sum_{j=1}^{i} \int_{0}^{w} \int_{0}^{z_{j}} q(t) h(t) d t d F_{\mathrm{II}}\left(z_{j}\right)\right\} \\
& =\left\{\int_{0}^{w} \int_{0}^{x} q(t) h(t) d t d F_{\mathrm{II}}(x)\right\} \sum_{i=1}^{\infty} i \operatorname{Pr}\{N(w)=i\} \\
& =\left\{\int_{0}^{w} \int_{0}^{x} q(t) h(t) d t d F_{\mathrm{II}}(x)\right\} \sum_{i=1}^{\infty} F_{\mathrm{II}}^{(i)}(w) \\
& =M(w) \int_{0}^{w} \int_{0}^{x} q(t) h(t) d t d F_{\mathrm{II}}(x)
\end{aligned}
$$

- The number of type I failures in the interval $\left(S_{N_{w}}, w\right)$ is $\int_{0}^{w} \int_{0}^{y} q(t) h(t) d t d H_{w}(y)$,

- If no type II failure occurs within time interval $(0, w)$, the number of type I failures in the interval $(0, w)$ is $\left(1-F_{\mathrm{II}}(w)\right) \int_{0}^{w} q(t) h(t) d t$, and

- The number of type I failures in the interval $\left(w, t_{d}\right)$ is $\bar{G}_{w}\left(t_{0}\right) \int_{0}^{t_{0}} h_{\mathrm{I}}(t) d t+\int_{0}^{t_{0}} h_{\mathrm{I}}(t) d G_{w}(t)$.

The numbers of type II failures can be estimated as follows.

- The number of type II failures in time interval $(0, w)$ is $M(w)$, and

- the number of type II failures in time interval $\left(w, t_{d}\right)$ is $G_{w}\left(t_{0}\right)$.

To summarise up the above five bulletined items, we can have the expected value of $C_{f}$ given by

$$
\begin{aligned}
E\left[C_{f}\right] & =c_{f} M(w) \int_{0}^{w} \int_{0}^{x} q(t) h(t) d t d F_{\mathrm{II}}(x) \\
& +c_{f}\left(\int_{0}^{w} \int_{0}^{y} q(t) h(t) d t d H_{w}(y)+\bar{G}_{w}\left(t_{0}\right) \int_{0}^{t_{0}} h_{\mathrm{I}}(t) d t+\int_{0}^{t_{0}} h_{\mathrm{I}}(t) d G_{w}(t)\right) \\
& +c_{f}\left(1-F_{\mathrm{II}}(w)\right) \int_{0}^{w} q(t) h(t) d t+c_{f}\left(M(w)+G_{w}\left(t_{0}\right)\right)
\end{aligned}
$$


Proof of Lemma 2. For Eq. (14), let $\frac{\partial D\left(k_{0}, t_{d}\right)}{\partial t_{d}}=0$, this gives

$$
\left(c_{\mathrm{I}}+c_{f}\right) h\left(t_{d}\right) t_{d}-C_{0}-k c_{e}-c_{d}-c_{\mathrm{I}} \int_{w}^{t_{d}} h(t) d t-c_{f} \int_{0}^{t_{d}} h(t) d t=0
$$

Denote the right-hand of Eq. $(23)$ by $L_{1}\left(k_{0}, t_{d}\right)$. then we have

- If Eq. (18) is satisfied, $L_{1}\left(k_{0}, w\right)<0$.

- As $h(t)$ is a non-decreasing positive function, $\int_{w}^{t_{d}} h(t) d t<h\left(t_{d}\right) t_{d}$ and $\int_{0}^{t_{d}} h(t) d t<h\left(t_{d}\right) t_{d}$. If $t \rightarrow \infty$, then $L_{1}\left(k_{0}, \infty\right)>0$.

Hence there is one value $w<t_{d}^{*}<\infty$ that satisfies Eq. (23), or then there exists one solution $t_{d}^{*}$ that minimises the life cycle average cost in Eq. (14).

Proof of Lemma 3. Let $\frac{\partial D\left(k_{0}, t_{d}\right)}{\partial t_{d}}=0$, that is

$$
\begin{aligned}
& \left(c_{\mathrm{II}}-c_{d}+c_{f}\right) g_{w}\left(t_{0}\right)\left(w+\int_{0}^{t_{0}}\left(1-G_{w}(t)\right) d t\right) \\
- & \left(1-G_{w}\left(t_{0}\right)\right)\left[C_{0}+k_{0} c_{e}+c_{d}+\left(c_{\mathrm{II}}-c_{d}\right) G_{w}\left(t_{0}\right)+c_{f}\left(M(w)+G_{w}\left(t_{0}\right)\right)\right] \\
= & 0
\end{aligned}
$$

where $g_{w}(0)=\frac{d G_{w}\left(t_{d}-w\right)}{d t_{d}}$ and $t_{0}=t_{d}-w$. Denote the right-hand of Eq. (24) by $L_{2}\left(k_{0}, t_{d}\right)$, then

- $L_{2}\left(k_{0}, w\right)=g_{w}(0)\left(c_{\mathrm{II}}-c_{d}+c_{f}\right) w-\left(C_{0}+k_{0} c_{e}+c_{d}+c_{f} M(w)\right)$. From Eq. (19), $L_{2}\left(k_{0}, w\right)<0$; and

- $L_{2}\left(k_{0}, \infty\right)=\left(c_{\mathrm{II}}-c_{d}+c_{f}\right) g_{w}(\infty)\left(w+\int_{0}^{\infty}\left(1-G_{w}(t)\right) d t\right)>0$.

Hence there is one value $w<t_{d}^{*}<\infty$ that satisfies Eq. (24), which minimises the life cycle average cost in Eq. (15).

Proof of Lemma 4. To find the optimal solution $t_{d}^{*}$ that minimises $D\left(k, t_{d}\right)=$ $\frac{C\left(k, t_{d}\right)}{B\left(k, t_{d}\right)}$, where $C\left(k, t_{d}\right)$ and $D\left(k, t_{d}\right)$ are from Eqs. (16) and (17), respectively. We set $\frac{d D\left(k, t_{d}\right)}{d t_{d}}=0$, which results in

$$
\frac{\left(c_{\mathrm{I}}+c_{f}\right) q_{0}}{1-q_{0}} \bar{G}_{w}\left(t_{0}\right)+\left(\beta_{2}+\left(c_{\mathrm{I}}+c_{f}\right) q_{0} \lambda\right) t_{0}+\beta_{3}+\frac{\left(c_{\mathrm{I}}+c_{f}\right) q_{0}}{1-q_{0}}=0 .
$$


Using the second order Taylor expansion of $\bar{G}\left(t_{0}\right)$ at $t_{0}=0$, we can have

$$
\beta_{1} t_{0}^{2}+\beta_{2} t_{0}+\beta_{3}=0
$$

Solve Eq. (26) and consider $t_{0}=t_{d}-w$, we can obtain the solution in Eq. (20). 